\title{
Nonlinear Dynamics for an Electromechanical Integrated Electrostatic Harmonic Drive
}

\author{
Lizhong Xu, Cuirong Zhu and Lei Qin \\ Mechanical Engineering Institute, Yanshan University, Qinhuangdao 066004, China
}

(Received 27 June 2005; revised 7 February 2006; accepted 21 April 2006)

In this paper, an electromechanically coupled dynamic equation for an integrated electrostatic harmonic drive is transformed into a balance equation for static displacement and a dynamic equation for dynamic displacement. By defining the electric field force in Fourier series form, static and dynamic electric field forces are presented. Mode functions and nonlinear dynamic equations for the drive system are introduced. Using Lindstent's perturbation method, nonlinear free vibration, forced response far from and near to natural frequency are discussed, respectively. Results show: The natural frequencies of the nonlinear drive system are smaller than those of the linear system. As the nonlinear parameter increases, the natural frequencies of the drive system decrease and the vibrating magnitude increases. When the exciting frequency is far from the natural frequency of the drive system, periodic times of the forced responses are all identical for different modes. When the exciting frequency is near to the natural frequency of the drive system, periodic times of the forced responses are different for different modes. The initial voltage and the clearance between the flexible ring and the outer stator have an obvious influence on the natural frequency and vibrating magnitude of the nonlinear drive system.

\section{INTRODUCTION}

The harmonic drive was invented by C. W. Musser. ${ }^{1-3}$ The drive can transmit large torque in a small size. The drive is suitable for applications in various technical fields, including aviation and space flight. Some countries have been developing the drive for several years..$^{4-9}$ In these studies, mesh theory, fatigue strength, dynamics and control, and manufacturing technique of the drive were developed. Based on the harmonic drive, the electromagnetic harmonic drive ${ }^{\mathbf{1 0}}$ and the harmonic piezodrive ${ }^{11}$ were proposed. In these two drives, the meshes between the flexible ring and the rigid ring are controlled by electromagnetic forces or the piezoactuator. In these two drives, integration of the harmonic drive and motor is realised.

Micro-electromechanical systems (MEMS) have potential applications in electronic assembly, medical, microspacecraft, and military equipment. The MEMS devices require high integration of the mechanical, electrical and control techniques. ${ }^{12-15}$ Based on the electromagnetic harmonic drive and the harmonic piezodrive, the authors have invented an active electromechanical integrated electrostatic harmonic drive (shown in Fig. 1). The drive consists principally of a flexible inner ring and a rigid outer stator ring. The latter are energised sequentially by voltages, and a rotational electric field results in a periodic elastic deformation of the flexible ring. This deformation is accompanied by periodic changes in the capacitance between the flexible ring and the rigid stator. These changes in capacitance produce tangential electric forces that cause the axis, on which the flexible ring is supported, to rotate. In the drive, integration of the harmonic drive, motor, and control is realised. This is a new concept for electromechanical drive systems.

Compared with piezoelectric and electromagnetic actuation principles, the electromechanical integrated electrostatic harmonic drive needs neither additional elements like coils or cores, nor special materials like piezoelectric ceramics. This is more favourable for miniaturisation of the electromechanical devices. Compared with other electrostatic actuation principles, ${ }^{16-18}$ the drive does not require fabrication of teeth on micro elements, and its rotational axis does not wobble. This makes it easier to be fabricated and used.

The output torque for an electrostatic wobble micromotor is calculated. ${ }^{16}$ In order to give a comparison of the loadcarrying capacity of the system invented by the authors with the electrostatic wobble micromotor, the authors calculated the output torque for an electrostatic harmonic actuator with approximately the same dimensions. The results show that the output torque of the system invented by the authors is larger than that of the electrostatic wobble micromotor.

Understanding the dynamic behaviour of MEMS is very important for controlling their performance. Dynamics about the micro ring subjected to electrostatic force has not been found yet. Only dynamics about the micro beam and plate subjected to electrostatic force has been found. ${ }^{19-21} \mathrm{~A}$ onedimensional (1-D) lumped model is used and the plate is considered at one point to vibrate. ${ }^{19,20}$ The micro beam is considered as a continuous body and the FEM method is used. ${ }^{21} \mathrm{Re}-$ sults show that the natural frequencies of a micro beam are in the order of $\mathrm{KHz}, \mathrm{MHz}$, or even $\mathrm{GHz}$ and various nonlinear behaviours are observed.

In this paper, an electromechanically coupled dynamic equation for the integrated electrostatic harmonic drive is transformed into a balance equation for static displacement and a dynamic equation for dynamic displacement. By defining the electric field force in a Fourier series form, the distribution of the static value and the dynamic value of the electric field force are presented. The mode functions and the nonlinear dynamic equation of the drive system are introduced. By using Lindstent's perturbation method, nonlinear free vibration, forced responses far from and near to natural frequencies can be examined. Here, the flexible micro-ring is considered as a continuous body and the analytic method is 